\title{
An Extension of Weighted Cumulative Inaccuracy Measure Based on Doubly Truncation
}

\author{
S. Jalayeri \\ Department of Statistics, University of Birjand, Iran \\ samirajalayeri@yahoo.com \\ M. Khorashadizadeh \\ Department of Statistics, University of Birjand, Iran \\ m.khorashadizadeh@birjand.ac.ir
}

\begin{abstract}
In the present communication, based on two extensions of Shannon entropy cumulative entropy by Rao, et al, 2004 and Kerridge's inaccuarcy by Kerridge, (1961), we study and obtain some properties of weighted cumulative residual (past) inaccuracy for truncated random variables. Several properties, including monotonicity, bounds and their connections to proportional (reversed) hazard model are obtained for left, right and doubly truncated random variables.
\end{abstract}

Keywords: Cumulative residual (past) inaccuracy, Inaccuracy measure, Interval cumulative residual inaccuracy, Weighted interval cumulative residual inaccuracy, Proportional (reversed) hazard model.

\section{Introduction and preliminary results}

In order to modelling the best probability density (mass) function based on the data given via information theory, many researchers have been introduced and studied some measures. One of these measure called "inaccuracy measure" has been introduced by Kerridge (1961) by,

$$
\kappa_{P, Q}=-\sum_{i=1}^{n} p_{i} \log q_{i}
$$

where $P=\left(p_{1}, \ldots, p_{n}\right)$ refers to true probabilities and $Q=\left(q_{1}, \ldots, q_{n}\right)$ refers to proposed experimenter probabilities. This measure has been extensively used as a useful tool for measurement of error in experimental results. Nath (1968) extended this measure to the case of continuous lifetime random variables by,

$$
\kappa_{X, Y}=-\int_{0}^{\infty} f(x) \log g(x) d x,
$$

where $f(x)$ is the actual density function corresponding to the observations and $g(x)$ is the density assigned by experimenter. As a special case when $P=Q$ or $f(x)=g(x)$, (4) and (2) reduces respectively to the well-known Shannon and differential Shannon entropies. Some properties, characterizations and extentions of the inaccuracy measure of form (2) have been obtained by many authors such as, Nair and Gupta (2007), Taneja et al. (2009), Kumar et al. (2011) and Kundu and Nanda (2015). As an alternative to Shannon entropy, Rao et al. (2004) have introduced cumulative entropy via replacing 
$f(x)$ by $\bar{F}(x)=1-F(x)$. This measure is valid in the continuous and discrete domains and have more advantages than Shannon entropy. In similar way of cumulative entropy, Taneja and Kumar (2012) have defined cumulative inaccuracy by

$$
c \kappa_{X, Y}=-\int_{0}^{\infty} \bar{F}(x) \log \bar{G}(x) d x
$$

On the other hand, in some applied problems such as reliability or mathematical neurobiology, we need to study the shift-dependent version of information measures. In this situation, Belis and Guiasu (1968) and Guiasu (1971), considered the notions of "weighted entropy" by

$$
H_{X}^{w}=-\sum_{i=1}^{n} w_{i} p_{i} \log p_{i}
$$

where $w_{i}$ are the weight of the elementary event. Similary, Taneja and Tuteja (1986), defined the weighted inaccuracy measure

$$
\kappa_{X, Y}^{w}=-\sum_{i=1}^{n} w_{i} p_{i} \log q_{i}
$$

which is a quantitative-qualitative measure of inaccuracy associated with the statement of an experimenter. In weighted measure the factor $w_{i} \geq 0$, may be viewed as a weight emphasizing the occurrence of the event $X=x_{i}$ which for greater $w_{i}$ yields a length biased shift-dependent information measure assigning greater importance to $x_{i}$. The inaccuracy and cumulative inaccuracy measures have been redefined based on residual lifetime, $X_{t}=(X-t \mid X \geq t)$, past lifetime, $X_{t}^{*}=(t-X \mid X \leq t)$ and interval lifetimes, $X_{t_{1}, t_{2}}=\left(X-t_{1} \mid t_{1} \leq X \leq t_{2}\right)$ and $X_{t_{1}, t_{2}}^{*}=\left(t_{2}-X \mid t_{1} \leq X \leq t_{2}\right)$. The history of these extentions and definitions are presented in Table 1.

One can see more results and properties of above measures in works by Prakash and Taneja (1986), Bhatia and Taneja (1991), Smitha(2010) and Kundu (2014). Analogous to weighted interval inaccuracy measure, definded by Kundu (2016), the following information measures can be considered.

Let $X$ and $Y$ be nonnegative lifetime random variables having distribution functions $F(x)$ and $G(x)$, reliability functions $\bar{F}(x)$ and $\bar{G}(x)$, respectively. Then, for any measurable function $\phi(x)$ (where $\phi(0)=0$ ) and based on random variables $X \mid t_{1}<X<t_{2} \quad$ and $Y \mid t_{1}<Y<t_{2} \quad$ where, $\quad\left(t_{1}, t_{2}\right) \in D:=\left\{(u, v) \in R^{2}+: F(u)<F(v)\right.$ and $G(u)<G(v)\}$ the weighted interval cumulative residual inaccuracy (WICRI) can be defined as

$$
c \kappa_{X, Y}^{w}\left(t_{1}, t_{2}\right)=-\int_{t_{1}}^{t_{2}} \phi(x) \frac{\bar{F}(x)}{\bar{F}\left(t_{1}\right)-\bar{F}\left(t_{2}\right)} \log \frac{\bar{G}(x)}{\bar{G}\left(t_{1}\right)-\bar{G}\left(t_{2}\right)} d x,
$$

and the weighted interval cumulative past inaccuracy (WICPI) is defined as, 


$$
c \kappa_{X, Y}^{* w}\left(t_{1}, t_{2}\right)=-\int_{t_{1}}^{t_{2}} \phi(x) \frac{F(x)}{F\left(t_{2}\right)-F\left(t_{1}\right)} \log \frac{G(x)}{G\left(t_{2}\right)-G\left(t_{1}\right)} d x .
$$

Table 1: Various definitions of inaccuracy measure

\begin{tabular}{|c|c|c|}
\hline Measure & Dynamic version & Interval version \\
\hline \multirow{2}{*}{$\begin{array}{c}\kappa_{X, Y}=-\int_{0} f(x) \log g(x) d x \\
\text { Nath (1968) }\end{array}$} & $\begin{array}{c}\kappa_{X, Y}(t)=-\int_{t}^{\infty} \frac{f(x)}{\bar{F}(t)} \log \frac{g(x)}{\bar{G}(t)} d x \\
\text { Taneja et al. (2009) }\end{array}$ & \multirow{2}{*}{$\begin{array}{l}\kappa_{X, Y}\left(t_{1}, t_{2}\right)= \\
-\int_{t_{1}}^{t_{2}} \frac{f(x)}{\bar{F}\left(t_{1}\right)-\bar{F}\left(t_{2}\right)} \log \frac{g(x)}{\bar{G}\left(t_{1}\right)-\bar{G}\left(t_{2}\right)} d x \\
\quad \text { Kundu and Nanda }(2015)\end{array}$} \\
\hline & $\begin{array}{r}\kappa_{X, Y}^{*}(t)=-\int_{0}^{t} \frac{f(x)}{F(t)} \log \frac{g(x)}{G(t)} d x \\
\text { Kumar et al. (2011) }\end{array}$ & \\
\hline \multirow{2}{*}{$\begin{array}{c}\kappa_{X, Y}^{w}=-\int_{0}^{\infty} x f(x) \log g(x) d x \\
\text { Kumar et al. (2010) }\end{array}$} & $\begin{array}{c}\kappa_{X, Y}^{w}(t)=-\int_{t}^{\infty} x \frac{f(x)}{\bar{F}(t)} \log \frac{g(x)}{\bar{G}(t)} d x \\
\text { Kumar et al. (2010) }\end{array}$ & \multirow{2}{*}{$\begin{array}{l}\kappa_{X, Y}^{w}\left(t_{1}, t_{2}\right)= \\
-\int_{t_{1}}^{t_{2}} x \frac{f(x)}{\bar{F}\left(t_{1}\right)-\bar{F}\left(t_{2}\right)} \log \frac{g(x)}{\bar{G}\left(t_{1}\right)-\bar{G}\left(t_{2}\right)} d x \\
\text { Kundu (2017) }\end{array}$} \\
\hline & $\begin{array}{c}\kappa_{X, Y}^{*_{W}}(t)=-\int_{0}^{t} x \frac{f(x)}{F(t)} \log \frac{g(x)}{G(t)} d x \\
\text { Kumar and Taneja (2012) }\end{array}$ & \\
\hline $\begin{array}{l}c \kappa_{X, Y}=-\int_{0}^{\infty} \bar{F}(x) \log \bar{G}(x) d x \\
\text { Taneja and Kumar (2012) }\end{array}$ & $\begin{array}{c}c \kappa_{X, Y}(t)=-\int_{t}^{\infty} \frac{\bar{F}(x)}{\bar{F}(t)} \log \frac{\bar{G}(x)}{\bar{G}(t)} d x \\
\text { Taneja and Kumar }(2012)\end{array}$ & $\begin{array}{l}c \kappa_{X, Y}\left(t_{1}, t_{2}\right)= \\
-\int_{t_{1}}^{t_{2}} \frac{\bar{F}(x)}{\bar{F}\left(t_{1}\right)-\bar{F}\left(t_{2}\right)} \log \frac{\bar{G}(x)}{\bar{G}\left(t_{1}\right)-\bar{G}\left(t_{2}\right)} d x \\
\text { Kundu (2016) }\end{array}$ \\
\hline $\begin{array}{c}c \kappa_{X, Y}^{*}=-\int_{0}^{\infty} F(x) \log G(x) d x \\
\text { Kundu (2016) }\end{array}$ & $\begin{array}{c}c \kappa_{X, Y}^{*}(t)=-\int_{0}^{t} \frac{F(x)}{F(t)} \log \frac{G(x)}{G(t)} d x \\
\text { Kundu (2016) }\end{array}$ & $\begin{array}{l}c \kappa_{X, Y}^{*}\left(t_{1}, t_{2}\right)= \\
-\int_{t_{1}}^{t_{2}} \frac{F(x)}{F\left(t_{2}\right)-F\left(t_{1}\right)} \log \frac{G(x)}{G\left(t_{2}\right)-G\left(t_{1}\right)} d x \\
\text { Kundu (2016) }\end{array}$ \\
\hline
\end{tabular}

The special cases of the above measures can be called as follow:

- For $t_{1}=0$ and $t_{2}=\infty$, we have weighted cumulative residual inaccuracy (WCRI) as,

$$
c \kappa_{X, Y}^{w}=-\int_{0}^{\infty} \phi(x) \bar{F}(x) \log \bar{G}(x) d x,
$$

and weighted cumulative past inaccuracy (WCPI),

$$
c \kappa_{X, Y}^{* w}=-\int_{0}^{\infty} \phi(x) F(x) \log G(x) d x .
$$


- For $t_{1}=t$ and $t_{2}=\infty$, we have weighted dynamic cumulative residual inaccuracy (WDCRI) as,

$$
c \kappa_{X, Y}^{w}(t)=-\int_{t}^{\infty} \phi(x) \frac{\bar{F}(x)}{\bar{F}(t)} \log \frac{\bar{G}(x)}{\bar{G}(t)} d x .
$$

- For $t_{1}=0$ and $t_{2}=t$, we have weighted dynamic cumulative past inaccuracy (WDCPI) as,

$$
c \kappa_{X, Y}^{* w}(t)=-\int_{0}^{t} \phi(x) \frac{F(x)}{F(t)} \log \frac{G(x)}{G(t)} d x
$$

In particular case of $\phi(x)=1$ the measures (6) to (11) yield the measures defined by Taneja and Kumar (2012) and Kundu et al. (2016) and in case of $\phi(x)=x$ we will have the definitions of length-biased version of the measures.

In the rest of the paper, we will obtain some properties of general measures WICRI, $c \kappa_{X, Y}^{w}\left(t_{1}, t_{2}\right)$, and WICPI, $c \kappa_{X, Y}^{* w}\left(t_{1}, t_{2}\right)$ and some remarks for special cases.

\section{Weighted interval cumulative residual (past) inaccuracy}

From now on, for given weight function $\phi(x)$ we will use the following notations,

$$
\begin{aligned}
& \phi^{\prime}(x)=\frac{\partial}{\partial x} \phi(x), \psi(x)=\int_{0}^{x} \phi(u) d u, \delta_{\phi}^{w}\left(t_{1}, t_{2}\right)=-\int_{t_{1}}^{t_{2}} \phi(x) \frac{\bar{F}(x)}{\bar{F}\left(t_{1}\right)-\bar{F}\left(t_{2}\right)} d x \text { and } \\
& \delta_{\phi}^{* w}\left(t_{1}, t_{2}\right)=-\int_{t_{1}}^{t_{2}} \phi(x) \frac{F(x)}{F\left(t_{2}\right)-F\left(t_{1}\right)} d x .
\end{aligned}
$$

Also, if $t_{1}=0$ and $t_{2}=\infty$ then $\delta_{\phi}^{w}=-\int_{0}^{\infty} \phi(x) \bar{F}(x) d x$, and if $t_{1}=t$ and $t_{2}=\infty$ then $\delta_{\phi}^{w}(t)=-\int_{t}^{\infty} \phi(x) \frac{\bar{F}(x)}{\bar{F}(t)} d x$.

One can rewritten equivalent form for IWCRE by using integral by parts as,

$$
\begin{aligned}
c \kappa_{X, Y}^{w}\left(t_{1}, t_{2}\right)= & -\frac{1}{\bar{F}\left(t_{1}\right)-\bar{F}\left(t_{2}\right)} \int_{t_{1}}^{t_{2}} \phi(x) \bar{F}(x) \log \bar{G}(x) d x+\delta_{\phi}^{w}\left(t_{1}, t_{2}\right) \log \left(\bar{G}\left(t_{1}\right)-\bar{G}\left(t_{2}\right)\right) \\
= & -\frac{1}{\bar{F}\left(t_{1}\right)-\bar{F}\left(t_{2}\right)} \int_{t_{1}}^{t_{2}} \phi(x) \bar{F}(x) \log \bar{G}(x) d x+\left\{\frac{\psi\left(t_{2}\right) \bar{F}\left(t_{2}\right)-\psi\left(t_{1}\right) \bar{F}\left(t_{1}\right)}{\bar{F}\left(t_{1}\right)-\bar{F}\left(t_{2}\right)}\right. \\
& \left.+E\left(\psi(X) \mid t_{1}<X<t_{2}\right)\right\} \log \left(\bar{G}\left(t_{1}\right)-\bar{G}\left(t_{2}\right)\right) .
\end{aligned}
$$

Also for WICPI we get,

$c \kappa_{X, Y}^{* w}\left(t_{1}, t_{2}\right)=-\frac{1}{F\left(t_{2}\right)-F\left(t_{1}\right)} \int_{t_{1}}^{t_{2}} \phi(x) F(x) \log G(x) d x+\delta_{\phi}^{* w}\left(t_{1}, t_{2}\right) \log \left(G\left(t_{2}\right)-G\left(t_{1}\right)\right)$ 


$$
\begin{aligned}
= & -\frac{1}{F\left(t_{2}\right)-F\left(t_{1}\right)} \int_{t_{1}}^{t_{2}} \phi(x) F(x) \log G(x) d x+\left\{\frac{\psi\left(t_{2}\right) F\left(t_{2}\right)-\psi\left(t_{1}\right) F\left(t_{1}\right)}{F\left(t_{2}\right)-F\left(t_{1}\right)}\right. \\
& \left.-E\left(\psi(X) \mid t_{1}<X<t_{2}\right)\right\} \log \left(G\left(t_{2}\right)-G\left(t_{1}\right)\right) .
\end{aligned}
$$

Now if $t_{1}=0$ and $t_{2}=\infty$ then $c \kappa_{X, Y}^{w}=-\int_{0}^{\infty} \phi(x) \bar{F}(x) \log \bar{G}(x) d x$ and also if $t_{1}=t$ and $t_{2}=\infty$ then

$c \kappa_{X, Y}^{w}(t)=-\frac{1}{\bar{F}(t)} \int_{t}^{\infty} \phi(x) \bar{F}(x) \log \bar{G}(x) d x+E(\psi(X)-\psi(t) \mid t<X<\infty) \log \bar{G}(t)$.

Or in relation with reliability measures, the WICRI can be rewrite as follow,

$$
\begin{aligned}
c \kappa_{X, Y}^{w}\left(t_{1}, t_{2}\right)= & -\int_{t_{1}}^{t_{2}} \int_{0}^{x} \phi^{\prime}(y) \frac{\bar{F}(x)}{\bar{F}\left(t_{1}\right)-\bar{F}\left(t_{2}\right)} \log \frac{\bar{G}(x)}{\bar{G}\left(t_{1}\right)-\bar{G}\left(t_{2}\right)} d y d x \\
= & -\int_{t_{1}}^{t_{2}} \int_{0}^{t_{1}} \phi^{\prime}(y) \frac{\bar{F}(x)}{\bar{F}\left(t_{1}\right)-\bar{F}\left(t_{2}\right)} \log \frac{\bar{G}(x)}{\bar{G}\left(t_{1}\right)-\bar{G}\left(t_{2}\right)} d y d x \\
& -\int_{t_{1}}^{t_{2}} \int_{y}^{t_{2}} \phi^{\prime}(y) \frac{\bar{F}(x)}{\bar{F}\left(t_{1}\right)-\bar{F}\left(t_{2}\right)} \log \frac{\bar{G}(x)}{\bar{G}\left(t_{1}\right)-\bar{G}\left(t_{2}\right)} d x d y \\
= & \phi\left(t_{1}\right) c \kappa_{X, Y}\left(t_{1}, t_{2}\right)-\int_{t_{1}}^{t_{2}} \phi^{\prime}(x) \frac{\bar{F}(x)-\bar{F}\left(t_{2}\right)}{\bar{F}\left(t_{1}\right)-\bar{F}\left(t_{2}\right)}\left\{-c \kappa_{X, Y}\left(x, t_{2}\right)\right. \\
& +\log \frac{\bar{G}(x)-\bar{G}\left(t_{2}\right)}{\bar{G}\left(t_{1}\right)-\bar{G}\left(t_{2}\right)} \times\left(\mu\left(x, t_{2}\right)+\frac{\left(t_{2}-x\right) \bar{F}\left(t_{2}\right)}{\left.\bar{F}\left(t_{1}\right)-\bar{F}\left(t_{2}\right)\right\} d x,}\right.
\end{aligned}
$$

where $\mu(x, y)=\frac{(y-x) \bar{F}(x)-\int_{x}^{y} \bar{F}(t) d t}{\bar{F}(x)-\bar{F}(y)}$ is the doubly truncated mean residual life and similarly

$$
\begin{aligned}
c \kappa_{X, Y}^{* w}\left(t_{1}, t_{2}\right) & =\phi\left(t_{1}\right) c \kappa_{X, Y}^{*}\left(t_{1}, t_{2}\right)-\int_{t_{1}}^{t_{2}} \phi^{\prime}(x) \frac{F\left(t_{2}\right)-F(x)}{F\left(t_{2}\right)-F\left(t_{1}\right)}\left\{-c \kappa_{X, Y}^{*}\left(x, t_{2}\right)\right. \\
+ & \left.\log \frac{G\left(t_{2}\right)-G(x)}{G\left(t_{2}\right)-G\left(t_{1}\right)} \times\left(\mu^{*}\left(x, t_{2}\right)-\frac{\left(x-t_{2}\right) F(x)}{F\left(t_{2}\right)-F\left(t_{1}\right)}\right)\right\} d x,
\end{aligned}
$$

where $\mu^{*}(x, y)=\frac{(x-y) F(x)+\int_{x}^{y} F(t) d t}{F(y)-F(x)}$ is the doubly truncated mean past lifetime which was first introduced by Khorashadizadeh et al. (2012).

Now if $t_{1}=0$ and $t_{2}=\infty$ then

$$
c \kappa_{X, Y}^{w}=-\int_{0}^{\infty} \phi^{\prime}(x) \bar{F}(x)\left\{-c \kappa_{X, Y}(x)+\mu(x) \log \bar{G}(x)\right\} d x,
$$


and also if $t_{1}=t$ and $t_{2}=\infty$ then

$$
c \kappa_{X, Y}^{w}(t)=\phi(t) c \kappa_{X, Y}(t)-\int_{t}^{\infty} \phi^{\prime}(x) \frac{\bar{F}(x)}{\bar{F}(t)}\left\{-c \kappa_{X, Y}(x)+\mu(x) \log \frac{\bar{G}(x)}{\bar{G}(t)}\right\} d x .
$$

In the next theorem, we study characterization problem for the proposed measures (6) and (7) under the proportional hazard model (PHM) and proportional reversed hazard model (PRHM). Under these two models, refer Cox (1959) and Gupta et al. (1998), the survival and distribution functions of two random lifetime variables are related by

$$
\bar{G}(x)=[\bar{F}(x)]^{\theta}, \theta>0,
$$

for PHM and by

$$
G(x)=[F(x)]^{\theta}, \theta>0,
$$

for PRHM.

Theorem 2.1 If $X$ and $Y$ are satisfying

- the proportional hazard model, then

$$
c \kappa_{X, Y}^{w}\left(t_{1}, t_{2}\right)=\theta I \xi^{w}\left(t_{1}, t_{2}\right)-K\left(t_{1}, t_{2} ; \theta\right) \delta_{\phi}^{w}\left(t_{1}, t_{2}\right) .
$$

- the proportional reversed hazard model, then

$$
c \kappa_{X, Y}^{* w}\left(t_{1}, t_{2}\right)=\theta I \xi^{* w}\left(t_{1}, t_{2}\right)-K^{*}\left(t_{1}, t_{2} ; \theta\right) \delta_{\phi}^{* w}\left(t_{1}, t_{2}\right)
$$

where $K\left(t_{1}, t_{2} ; \theta\right)=\log \frac{\left[\bar{F}\left(t_{1}\right)-\bar{F}\left(t_{2}\right)\right]^{\theta}}{\bar{F}^{\theta}\left(t_{1}\right)-\bar{F}^{\theta}\left(t_{2}\right)}$ and $K^{*}\left(t_{1}, t_{2} ; \theta\right)=\log \frac{\left[F\left(t_{2}\right)-F\left(t_{1}\right)\right]^{\theta}}{F^{\theta}\left(t_{2}\right)-F^{\theta}\left(t_{1}\right)}$ and also,

$$
I \xi^{w}(x, y)=\int_{x}^{y} \phi(u) \frac{\bar{F}(u)}{\bar{F}(x)-\bar{F}(y)} \log \frac{\bar{F}(u)}{\bar{F}(x)-\bar{F}(y)} d u
$$

and

$$
I \xi^{*^{*}}(x, y)=\int_{x}^{y} \phi(u) \frac{F(u)}{F(y)-F(x)} \log \frac{F(u)}{F(y)-F(x)} d u,
$$

are respectively interval weighted cumulative residual entropy (IWCRE) and interval weighted cumulative past entropy (IWCPE) (Yasaei Sekeh et al., 2015).

Proof: By replacing (12) and (13) into (6) and (7) respectively the required results are implies.

Remark 2.2 For two absolutely continuous non-negative random variables $X$ and $Y$ and weight function $\phi(x)=x$, in special case of Theorem 2.1,

- when $t_{1}=0, t_{2}=\infty$, we have $c \kappa_{X, Y}^{w}=\theta \xi^{w}$ and $c \kappa_{X, Y}^{*^{*} w}=\theta \xi^{* w}$,

- when $t_{1}=0, t_{2}=t$, we have $c \kappa_{X, Y}^{* w}(t)=\theta \xi^{* w}(t)$, 
- when $t_{1}=t, t_{2}=\infty$, we have $c \kappa_{X, Y}^{w}(t)=\theta \xi^{w}(t)$.

Example 2.3 Let $X$ have an exponential distribution with mean $\frac{1}{\beta}, \beta>0$, and $Y=X^{\frac{1}{2}}$. Therefore $Y$ have a Weibull distribution with parameters $\left(2, \frac{2}{\beta}\right)$ and $X$ and $Y$ are satisfying the proportional hazard model. Then,

$$
c \kappa_{X, Y}\left(t_{1}, t_{2}\right)=\frac{2}{\beta}+\frac{1}{\beta} \log \left(1-e^{2 \beta\left(t_{1}-t_{2}\right)}\right)+\frac{2\left(t_{1}-t_{2}\right) e^{-\beta t_{2}}}{e^{-\beta t_{1}}-e^{-\beta t_{2}}} ;\left(t_{1}, t_{2}\right) \in D .
$$

We observe that for fixed value $t_{2} \in(0, \infty),(16)$ is decreasing in $t_{1} \in(0, \infty)$. Now, assume $\phi(x)=e^{\alpha x}$, applying (6) yields the following expression:

$$
\begin{aligned}
c \kappa_{X, Y}^{w}\left(t_{1}, t_{2}\right) & =\frac{1}{(\beta-\alpha)\left(e^{-\beta t_{2}}-e^{-\beta t_{1}}\right)}\left\{2 \beta\left(t_{2} e^{(\alpha-\beta) t_{2}}-t_{1} e^{(\alpha-\beta) t_{1}}\right)\right. \\
& \left.+\frac{2 \beta}{(\alpha-\beta)}\left(e^{(\alpha-\beta) t_{2}}-e^{(\alpha-\beta) t_{1}}\right)+\left(e^{(\alpha-\beta) t_{2}}-e^{(\alpha-\beta) t_{1}}\right) \cdot \log \left(e^{-2 \beta t_{2}}-e^{-2 \beta t_{1}}\right)\right\} .
\end{aligned}
$$

Note that when $\alpha \rightarrow 0$ then $c \kappa_{X, Y}^{w}\left(t_{1}, t_{2}\right) \rightarrow c \kappa_{X, Y}\left(t_{1}, t_{2}\right)$. One can easily check that for given $t_{2} \in(0, \infty), c \kappa^{w}\left(t_{1}, t_{2}\right)$ is not monotone decreasing in $t_{1}$. This means, if the monotonicity property for ICRI is fulfilled then there is no guarantee WICRI is monotone as well.

\section{Bounds for the WICRI and WICPI}

In this section, we give several bounds for the WICRI and WICPI by using assertions established in previous sections. Let us start with another alternative representation for the WICRI and WICPI. Let for $\left(t_{1}, t_{2}\right) \in D, \tau_{i}\left(t_{1}, t_{2}\right)=\frac{\bar{G}\left(t_{i}\right)}{\bar{G}\left(t_{1}\right)-\bar{G}\left(t_{2}\right)}, i=1,2$ then

$$
\begin{aligned}
c \kappa_{X, Y}^{w}\left(t_{1}, t_{2}\right)= & -\int_{t_{1}}^{t_{2}} \phi(x) \frac{\bar{F}(x)}{\bar{F}\left(t_{1}\right)-\bar{F}\left(t_{2}\right)} \log \tau_{1}\left(x, t_{2}\right) d x \\
& -\int_{t_{1}}^{t_{2}} \phi(x) \frac{\bar{F}(x)}{\bar{F}\left(t_{1}\right)-\bar{F}\left(t_{2}\right)} \log \frac{\bar{G}(x)-\bar{G}\left(t_{2}\right)}{\bar{G}\left(t_{1}\right)-\bar{G}\left(t_{2}\right)} d x,
\end{aligned}
$$

in addition

$$
\begin{aligned}
c \kappa_{X, Y}^{w}\left(t_{1}, t_{2}\right)= & -\int_{t_{1}}^{t_{2}} \phi(x) \frac{\bar{F}(x)}{\bar{F}\left(t_{1}\right)-\bar{F}\left(t_{2}\right)} \log \tau_{2}\left(t_{1}, x\right) d x \\
& -\int_{t_{1}}^{t_{2}} \phi(x) \frac{\bar{F}(x)}{\bar{F}\left(t_{1}\right)-\bar{F}\left(t_{2}\right)} \log \frac{\bar{G}\left(t_{1}\right)-\bar{G}(x)}{\bar{G}\left(t_{1}\right)-\bar{G}\left(t_{2}\right)} d x .
\end{aligned}
$$


Using $\tau_{i}^{*}\left(t_{1}, t_{2}\right)=\frac{G\left(t_{i}\right)}{G\left(t_{2}\right)-G\left(t_{1}\right)}, i=1,2$, similar equations can be obtain for WICPI, $c \kappa_{X, Y}^{* w}\left(t_{1}, t_{2}\right)$. In the next theorem a lower bound is obtained for WICRI where the same bounds can be obtained for WICPI.

Theorem 3.1 Let $X$ and $Y$ are non-negative random variables, then for given weight function $\phi(x)$ we have,

- If $\tau_{1}\left(t_{1}, t_{2}\right)$ is decreasing in $t_{1}$, then

$$
c \kappa_{X, Y}^{w}\left(t_{1}, t_{2}\right) \geq-\delta_{\phi}^{w}\left(t_{1}, t_{2}\right) \log \tau_{1}\left(x, t_{2}\right)
$$

- If $\tau_{2}\left(t_{1}, t_{2}\right)$ is increasing in $t_{2}$, then

$$
c \kappa_{X, Y}^{w}\left(t_{1}, t_{2}\right) \geq-\delta_{\phi}^{w}\left(t_{1}, t_{2}\right) \log \tau_{2}\left(t_{1}, x\right) .
$$

Proof: The theorem can be proved by using the definitions.

The following theorem describes the relationship between interval Shannon entropy and the interval weighted cumulative residual entropy and we prove that the WICRI is exponentially larger than interval Shannon entropy.

Theorem 3.2 Let $X$ and $Y$ are two non-negative continuous random variables with cumulative distribution functions $F(t)$ and $G(t)$ respectively, then for given weight function $\phi(x)$ we have,

$$
c \kappa_{X, Y}^{w}\left(t_{1}, t_{2}\right) \geq \beta\left(t_{1}, t_{2}\right) \exp \left\{I H_{X}\left(t_{1}, t_{2}\right)\right\}
$$

where $I H_{X}\left(t_{1}, t_{2}\right)=-\int_{t_{1}}^{t_{2}} \frac{f(x)}{\bar{F}\left(t_{1}\right)-\bar{F}\left(t_{2}\right)} \log \frac{f(x)}{\bar{F}\left(t_{1}\right)-\bar{F}\left(t_{2}\right)} d x$ is the interval Shannon entropy (Misagh and Yari, 2011;2012) and

$$
\beta\left(t_{1}, t_{2}\right)=\exp \left\{\frac{1}{\bar{F}\left(t_{1}\right)-\bar{F}\left(t_{2}\right)} \int_{\bar{F}\left(t_{2}\right)}^{\bar{F}\left(t_{1}\right)} \log \left\{-\phi\left(\bar{F}^{-1}(y)\right) \frac{y}{\bar{F}\left(t_{1}\right)-\bar{F}\left(t_{2}\right)} \log \frac{\bar{G}\left(\bar{F}^{-1}(y)\right)}{\bar{G}\left(t_{1}\right)-\bar{G}\left(t_{2}\right)}\right\} d y\right\} .
$$

Proof: In log-sum inequality of form,

$$
\int_{B} a(x) \ln \frac{a(x)}{b(x)} d x \geq \int_{B} a(x) d x\left(\log \frac{\int_{B} a(x) d x}{\int_{B} b(x) d x}\right), \quad a(x)>0, b(x)>0,
$$

if we put, $a(x)=\frac{f(x)}{\bar{F}\left(t_{1}\right)-\bar{F}\left(t_{2}\right)}, b(x)=-\phi(x) \frac{\bar{F}(x)}{\bar{F}\left(t_{1}\right)-\bar{F}\left(t_{2}\right)} \log \frac{\bar{G}(x)}{\bar{G}\left(t_{1}\right)-\bar{G}\left(t_{2}\right)}$ and $B=\left(t_{1}, t_{2}\right)$ then we have 


$$
\begin{aligned}
& \int_{t_{1}}^{t_{2}} \frac{f(x)}{\bar{F}\left(t_{1}\right)-\bar{F}\left(t_{2}\right)} \log \frac{f(x)}{\bar{F}\left(t_{1}\right)-\bar{F}\left(t_{2}\right)} d x \\
& -\int_{t_{1}}^{t_{2}} \frac{f(x)}{\bar{F}\left(t_{1}\right)-\bar{F}\left(t_{2}\right)} \log \left\{-\phi(x) \frac{\bar{F}(x)}{\bar{F}\left(t_{1}\right)-\bar{F}\left(t_{2}\right)} \log \frac{\bar{G}(x)}{\bar{G}\left(t_{1}\right)-\bar{G}\left(t_{2}\right)}\right\} d x \\
& \geq-\log \left\{\int_{t_{1}}^{t_{2}} \phi(x) \frac{\bar{F}(x)}{\bar{F}\left(t_{1}\right)-\bar{F}\left(t_{2}\right)} \log \frac{\bar{G}(x)}{\bar{G}\left(t_{1}\right)-\bar{G}\left(t_{2}\right)} d x\right\} \\
& =\log \frac{1}{c \kappa_{X, Y}^{w}\left(t_{1}, t_{2}\right)},
\end{aligned}
$$

which is the required results.

Remark 3.3 The similar arguments for WICPI is achieved. In other words, owing to the definition of $I H_{X}\left(t_{1} ; t_{2}\right)$ we have,

$$
c \kappa_{X, Y}^{* w}\left(t_{1}, t_{2}\right) \geq \beta^{*}\left(t_{1}, t_{2}\right) \exp \left\{I H_{X}\left(t_{1}, t_{2}\right)\right\},
$$

where

$$
\beta^{*}\left(t_{1}, t_{2}\right)=\exp \left\{\frac{1}{F\left(t_{2}\right)-F\left(t_{1}\right)} \int_{F\left(t_{1}\right)}^{F\left(t_{2}\right)} \log \left\{-\phi\left(F^{-1}(y)\right) \frac{y}{F\left(t_{2}\right)-F\left(t_{1}\right)} \log \frac{G\left(F^{-1}(y)\right)}{G\left(t_{2}\right)-G\left(t_{1}\right)}\right\} d y\right\} .
$$

Remark 3.4 In special case of $t_{1}=0$ and $t_{2}=\infty$, the Theorem 3.2 leads to

$$
c \kappa_{X, Y}^{w}(t) \geq \beta(t) \exp \left\{H_{X}(t)\right\}
$$

where $H_{X}(t)=-\int_{t}^{\infty} \frac{f(x)}{\bar{F}(t)} \log \frac{f(x)}{\bar{F}(t)} d x$ and

$$
\beta(t)=\exp \left\{\frac{1}{\bar{F}(t)} \int_{0}^{\bar{F}(t)} \log \left\{-\phi\left(\bar{F}^{-1}(y)\right) \frac{y}{\bar{F}(t)} \log \frac{\bar{G}\left(\bar{F}^{-1}(y)\right)}{\bar{G}(t)}\right\} d y\right\} .
$$

Remark 3.5 For two absolutely continuous non-negative random variables $X$ and $Y$ and weight function $\phi(x)=x$,

$$
c \kappa_{a X+b, a Y+b}^{w}\left(t_{1}, t_{2}\right)=a^{2} c \kappa_{X, Y}^{w}\left(\frac{t_{1}-b}{a}, \frac{t_{2}-b}{a}\right)+a b . c \kappa_{X, Y}\left(\frac{t_{1}-b}{a}, \frac{t_{2}-b}{a}\right) .
$$

Theorem 3.6 The WICRI is increasing (decreasing)

- with respect to $t_{1}$ if and only iffor $\left(t_{1}, t_{2}\right) \in D$,

$$
c \kappa_{X, Y}^{w}\left(t_{1}, t_{2}\right) \geq(\leq) \frac{h_{1}^{Y}\left(t_{1}, t_{2}\right)}{h_{1}^{X}\left(t_{1}, t_{2}\right)} \delta_{\phi}^{w}\left(t_{1}, t_{2}\right)-\frac{\phi\left(t_{1}\right)}{h_{X}\left(t_{1}\right)} \log \frac{h_{1}^{Y}\left(t_{1}, t_{2}\right)}{h_{Y}\left(t_{1}\right)},
$$

- with respect to $t_{2}$ if and only if for $\left(t_{1}, t_{2}\right) \in D$,

$$
c \kappa_{X, Y}^{w}\left(t_{1}, t_{2}\right) \leq(\geq) \frac{h_{2}^{Y}\left(t_{1}, t_{2}\right)}{h_{2}^{X}\left(t_{1}, t_{2}\right)} \delta_{\phi}^{w}\left(t_{1}, t_{2}\right)-\frac{\phi\left(t_{2}\right)}{h_{X}\left(t_{2}\right)} \log \frac{h_{2}^{Y}\left(t_{1}, t_{2}\right)}{h_{Y}\left(t_{2}\right)},
$$


where $h_{i}^{X}\left(t_{1}, t_{2}\right)=\frac{f\left(t_{i}\right)}{\bar{F}\left(t_{1}\right)-\bar{F}\left(t_{2}\right)}, i=1,2$ are the generalized failure rate (Navarro and Ruiz, 1996) and $h_{X}(t)=\frac{f(t)}{\bar{F}(t)}$ is the failure rate function.

Proof: Differentiating WICRI of form (6) with respect to $t_{1}$ and $t_{2}$ and doing some algebraic calculation the inequalities (21) and (22) yields. Similar results of Theorem 3.6 can be obtained for WICPI.

Remark 3.7 In special case of Theorem 3.6, when, we have,

$$
c \kappa_{X, Y}^{w}(t) \geq(\leq) \frac{h_{Y}(t)}{h_{X}(t)} \delta_{\phi}^{w}(t) .
$$

\section{Acknowledgments}

The authors thank the Editor-in-Chief of the journal and anonymous reviewers for their valuable comments and suggestions on the earlier version of the manuscript which lead to considerable improvement in the contents.

\section{References}

1. Bhatia, P.K. and Taneja, H.C. (1991). On characterization of quantitativequalitative measure of inaccuracy. Information Sciences, 56, 143-149.

2. Cox, D.R. (1959). The analysis of exponentially distributed lifetimes with two types of failures. Journal of the Royal Statistical Society: Series B, 21, 411-421.

3. Guiaşu, S. (1971). Weighted entropy. Reports on Mathematical Physics, 2(3), 165-179.

4. Gupta, R.C., Gupta P.L. and Gupta, R.D. (1998). Modelling failure time data by Lehman alternatives. Communications in Statistics- Theory and Methods, 27(4), 887-904.

5. Kerridge, D. F. (1961). Inaccuracy and inference. Journal of the Royal Statistical Society. Series B (Methodological), 184-194.

6. Khorashadizadeh, M., Rezaei Roknabadi, A. H., and Mohtashami Borzadaran, G. R. (2012). Characterizations of lifetime distributions based on doubly truncated mean residual life and mean past to failure. Communications in Statistics-Theory and Methods, 41(6), 1105-1115.

7. Kumar, V. and Taneja, H.C. (2012). On length biased dynamic measure of past inaccuracy. Metrika, 75, 73-84.

8. Kumar, V., Taneja, H.C. and Srivastava, R. (2010). Length biased weighted residual inaccuracy measure. Metron, LXVIII, 153-160.

9. Kumar, V., Taneja, H.C. and Srivastava, R. (2011). A dynamic measure of inaccuracy between two past lifetime distributions. Metrika, 74(1), 1-10. 
10. Kundu, C., Di Crescenzo, A. and Longobardi, M. (2016), On cumulative residual (past) inaccuracy for truncated random variables. Metrika, Vol 79 (3), pp 335356.

11. Kundu, C. (2014). Characterizations based on length-biased weighted measure of inaccuracy for truncated random variables. Applications of Mathematics, 59 (6), 697-714.

12. Kundu, C. (2017). On weighted measure of inaccuracy for doubly truncated random variables. Communications in Statistics-Theory and Methods, 46(7), 3135-3147.

13. Kundu, C. and Nanda, A. K. (2015). Characterizations based on measure of inaccuracy for truncated random variables. Statistical Papers, 56 (3), 619-637.

14. Misagh, F. and Yari, G.H. (2011), On weighted interval entropy. Statistics and Probability Letters, 81, 188-194.

15. Misagh, F. and Yari, G.H. (2012), Interval entropy and informative distance. Entropy, 14, 480-490.

16. Nair, N. U., and Gupta, R. P. (2007). Characterization of proportional hazard models by properties of information measures. International Journal of Statistical Sciences, 6, 223-231.

17. Nath, P. (1968). Inaccuracy and coding theory. Metrika, 13(1), 123-135.

18. Navarro, J. and Ruiz, J. M. (1996). Failure-rate function for doubly-truncated random variables. IEEE Trans. Reliab. 45(4), 685-690.

19. Prakash, O. and Taneja, H.C. (1986). Characterization of the quantitativequalitative measure of inaccuracy for discrete generalized probability distributions. Communications in Statistics- Theory and Methods, 15(12), 37633771.

20. Rao, M., Chen, Y., Vemuri, B. C. and Wang, F. (2004). Cumulative residual entropy: A new measure of infromation. IEEE Trans. Information Theory, 50, 6, 1220-1228.

21. Smitha, S. (2010). A Study on the Kerridge's Inaccuracy Measure and Related Concepts, Doctoral dissertation, Cochin University of Science and Technology.

22. Taneja, H. C., and Tuteja, R. K. (1986). Characterization of a quantitativequalitative measure of inaccuracy. Kybernetika, 22(5), 393-402.

23. Taneja, H. C., and Kumar, V. (2012). On dynamic cumulative residual inaccuracy measure. In Proceedings of the World Congress on Engineering, Vol. 1, 153-156.

24. Taneja, H.C., Kumar, V. and Srivastava, R. (2009). A dynamic measure of inaccuracy between two residual lifetime distributions. International Mathematical Forum, 4(25), 1213-1220.

25. Yasaei Sekeh, S. Mohtashami Borzadran, G.R and Rezaei Roknabadi, A.H. (2015). A note on double truncated (interval) weighted cumulative entropies. arXiv preprint arXiv:1508.00246. 